\title{
PHYSIOLOGICAL AND CLINICAL ASPECTS OF SOME RECENT WORK ON THE CARDIO-VASCULAR SYSTEM
}

By Professor O. G. Edholm, M.R.C.S., L.R.C.P.

Department of Physiology and Biochemistry Royal Veterinary College and Hospital, London, N.W.I

The close relationship between physiological research and progress in clinical work is self evident. Nevertheless there are certain difficulties in assessing the clinical applications of recent advances in physiology. It is not possible to cover the whole field of physiology and discuss the possible clinical implications ; impossible because of lack of knowledge and lack of space. This article has, therefore, been limited to such aspects as are familiar to the writer, those problems concerned with the cardio-vascular system. It is not suggested that advances in this field are amongst the most important, other physiologists would almost certainly select quite different examples with no doubt greater cogency.

In general it is true that there is a considerable time lag between a physiological discovery and its application to clinical medicine. This is not necessarily due to the reluctance of clinicians to apply physiology to medicine (although this may be a factor) or due to ignorance of advances in physiology. There are more serious difficulties, primarily that it is rare to find an immediate obvious clinical development of physiological work. This is probably the reason why many clinicians feel that it is a waste of time to keep up to date in their physiology. Critics ignore the fact that the study of physiology is not undertaken only to help in the solution of clinical problems, but is a subject worthy of study in its own right. This raises the old problem of the distinction between pure and applied research. There is no true difference, it is essentially a question of time, the applied work of today being based on the pure research of the past. The time lag is sometimes very short, e.g. Minot's successful treatment of pernicious anaemia following Weed's work on the liver, but usually there is a long delay. It is quite probable that these delays will be worse in the future. The distinction between clinical work and physiology becomes greater with increased specialization. In the past many of the active members of the
Physiological Society were physicians who not only listened, but contributed. This is unusual today; and the two sciences will become completely separated unless the gap is filled. An intermediary is needed between the physiologist and the clinician, someone who has interests on both sides, the man working on applied physiology.

Developments during the war have shown that the problems of applied physiology are not only of great practical importance but frequently are of real theoretical interest. Many physiologists have had to work on problems specifically those of war, e.g. on poison gases, problems which are not apparently directly related to those occurring in civil life. But there has also been very active research on such problems as the effects of high and low environmental temperature on the human body, the effects of gravity as illustrated by the high accelerations of modern aircraft, of anoxia, high pressure oxygen and so on. Much of this work will undoubtedly have a clinical application. The war therefore has stimulated interest in applied physiology, and it is to be hoped that this interest will be continued in peace time.

It should be clear that it is not very useful to try and discuss the clinical applications of recent physiological work as such discussion would be highly speculative. For example, in the field of cardio-vascular physiology some of the most striking work of recent years has been that on the foetal circulation. New and ingenious methods have been adopted and our knowledge both of the circulation of the foetus in utero and of the changes occurring in the circulation at birth has been greatly extended. ${ }^{2}$ But try and apply this work to clinical problems. All one can say is that we may be able to interpret the dynamics of congenital heart disease more skilfully. But there can be little doubt that eventually this work will be of the greatest practical importance: At present there are only vague possibilities. The work 
of the applied physiologist in the field of experimental medicine is of more interest and can be linked to the original work in pure physiology.

In the applied field, interest has begun to centre on recent developments in the technique of measuring cardiac output. Such measurements have been and will be of the greatest value in studying cardiac disease. Many of the methods used to measure cardiac output are based on the principle, first enunciated by Fick in 1870 . This states that the cardiac output (C.O.) can be calculated from the oxygen consumption of the subject and the arteriovenous difference in oxygen content of the $\mathrm{O}$

blood. C.O. $=\frac{}{\mathrm{A}-\mathrm{V}}$ (the carbon dioxide output and $\mathrm{A}-\mathrm{V}$ difference can equally well be used). The great difficulty of applying the Fick principle in man has always been the problem of collecting samples of mixed venous blood, i.e. of blood having the same oxygen and carbon dioxide content as that entering the pulmonary circulation. It is no use obtaining samples of venous blood from a peripheral vein, the gas contents are not necessarily representative, being dependent on the metabolism of, and rate of blood flow through, the part drained by that particular vein. Satisfactory, samples can only be obtained from the right auricle or right ventricle. In animals this can be done by direct cardiac puncture. Although this technique has been employed in man (in Germany) most people would justifiably hesitate before drawing blood direct in this way. Many ingenious methods have been developed to try and get over this difficulty. The equilibration of gas mixtures in the lungs with the venous blood is one such method. Hence by knowing the composition of the alveolar air which is found to be in equilibrium with the venous blood, the oxygen and carbon dioxide content of this blood can be calculated. Other methods involve the inhalation of acetylene or carbon monoxide. It is unnecessary to discuss in detail the technical problems involved in all of these methods. Valuable information has been obtained by their use, but they all require great skill on the part of the operator and considerable training of the subject. For these reasons the various indirect Fick methods have only a limited clinical application.

The introduction of the technique of cardiac catheterization is, therefore, of first-rate importance. The technique and the results obtained are fully described in recent publications by Cournand ${ }^{4}$ and his colleagues in America and McMichael and Sharpey-Schafer ${ }^{8}$ in this country. Briefly, the method consists of the introduction of a non-wettable X-ray opaque ureteric catheter into either the left or right antecubital vein. The catheter is pushed on via the vein into the right auricle, its position being checked by screening. Not only can samples of right auricular blood be withdrawn via the catheter but it is also possible to measure the venous pressure in the right auricle by connecting the catheter to a manometer, filled with heparinized saline. In practice a steady drip "of heparin saline is maintained between readings of venous pressure and the taking of blood samples, so preventing the formation of a thrombus at the tip of the catheter. Cardiac catheterization usually seems a dangerous and alarming procedure to those who are unfamiliar with it. However more than $\mathbf{I}, 800$ catheterizations have now been carried out in man without accidents which could be attributed to the catheter. It is, in the right hands, perfectly safe and its use has been overwhelmingly justified.

In order to discuss the results that have been obtained, it is necessary to refer back to physiological work. As long ago as 1910-15 Starling and his associates worked out the fundamental principles of cardiac dynamics, using a highly simplified version of the circulation, the heart-lung preparation. In this, nervous influences could be excluded, venous return controlled and the peripheral resistance to the outflow of blood from the heart adjusted within wide limits. The various experimental findings were brought together by Starling when he formulated his famous law of the heart; ' the larger the diastolic volume of the heart, the greater the energy of its contraction.' Like all physiological principles this was shown to be true only within limits. As soon as the diastolic volume fell very low or rose above a critical value the heart failed. In the intact animal measurement of diastolic 
volume is not easy. Starling showed, however, that there was a very important relationship between cardiac output and venous pressure as measured in the right auricle. As the venous pressure rose the cardiac output increased up to a maximum ; but a further rise of venous pressure was associated with a fall in cardiac output. ${ }^{9}$

The direct application of these results to man could not be said to be certain, without further experiment. Much animal work showed that Starling's conclusions were valid in the intact animal, even though they were not exclusive. In particular it was shown that as the venous return increased so the pressure in the right auricle rose.

It has been difficult to obtain evidence in man. For many years it has been known that venesection in congestive heart failure may be beneficial. This has been explained on the grounds that the heart has been distended, and that venesection relieved the distension. This explanation is superficial and it is reasonable to say that although the value of venesection was not in doubt detailed knowledge of the effects of venesection was lacking.

The study of cardiac output in man, both in health and disease has clearly demonstrated the relationship of right auricular pressure and. cardiac output. McMichael and SharpeySchafer ${ }^{6}$ were able to show that the data obtained by Starling in the heart-lung preparation was valid for man. In congestive heart failure the right auricular pressure is high and the cardiac output is low. Venesection reduces the right auricular pressure and the cardiac output increases. In normal subjects the right auricular pressure is also lowered by venesection but the cardiac output falls. The explanation of the benefit of venesection is now clear. In congestive failure the venous filling pressure is very high, so high that it is beyond the physiological optimum, i.e. over the crest of the curve and the heart is in fact overstretched. As a result the cardiac output is low. When the pressure in the right auricle is reduced the cardiac output increases, and it is this increase in the cardiac output which, in part at any rate, is responsible for the improvement in the condition of the patient. The normal heart is working on the rising side of the curve, and here a fall in V.P. = fall in C.O.
The effect of venesection and of haemorrhage has been studied in other conditions. The effects of haemorrhage has been the object of innumerable animal experiments. It might well be thought that on this subject we were reasonably well informed. On the contrary work on man has produced some totally unexpected findings which could not have been predicted from animal work. Following repeated haemorrhage, e.g. in cases of peptic ulcer, a severe anaemia with a low blood volume and a low haemoglobin develops. The cardiac output in these cases has been measured, and is found to be not decreased, but greatly increased. Further the right auricular pressure is raised.10 At first sight this is a surprising observation, but it is, in fact, essential for the survival of the individual that the cardiac output should be increased under these conditions. With a low haemoglobin and a low blood volume, the total oxygen capacity of the blood is greatly reduced. The oxygen requirements of the body will not be diminished so it follows that there are only two mechanisms for maintaining an oxygen supply. There may be increased reduction of haemoglobin in the tissues and the rate of blood flow round the body may be increased.? There is a definite limit to the degree of reduction of haemoglobin that is possible, and in these cases it is found that the blood from the right auricular is not greatly reduced. So the main compensatory mechanism is in fact an increased cardiac output.

There are several important consequences of this finding, clinical and physiological. Clinically, the obvious treatment of anaemia is blood transfusion, but this may be disastrous unless certain precautions are taken. When a blood transfusion is given the right auricular pressure rises and, in normal subjects, there is an increased cardiac output. But in chronic anaemia, the cardiac output is already very high and the right auricular pressure is raised. When blood is transfused into such a subject the right auricular pressure rises still higher and cardiac failure may ensue. So when a blood transfusion is given, packed red cells, not whole blood, are used and given very slowly and cautiously as if a powerful drug were being injected. In this way raising the right auricular pressure may be avoided. 
A second clinical point is that in severe anaemia cardiac failure may result, not directly due to the anaemia but owing to the long maintained high resting cardiac output.

The physiologist is interested in the mechanisms by which this hyperdynamic phase is brought about. The immediate effects of haemorrhage are a fall in right auricular pressure and a diminished cardiac output. At some time after a severe haemorrhage the change over takes place from the hypodynamic to the hyperdynamic phase. The mechanisms involved which bring about this change are at present unknown. But it is clear that in considering the adjustments of the circulation to haemorrhage, these long term effects which may take several days are as important as the better known immediate reactions. The existence of this problem could hardly have been discovered except by clinical work, since the bulk of animal work is acute, and covers only a short time period.

So much for the delayed effects of haemorrhage. There have also been some curious findings on the early effects, which emphasize the differences between man and animals. Many different measurements have been made on subjects after bleeding; $\mathrm{Hb}$. values, blood potassium, urine flow, etc. Peripheral blood flow has also been studied by means of the plethysmograph, an instrument which has a long history. Lewis and Grant are chiefly responsible for the design used in this country. Both in America and in this country the peripheral circulation is and has been studied in health and disease with the plethysmograph. Abramson has recently published a monograph reviewing the results obtained $^{1}$.

By means of the plethysmograph the changes which occur in the peripheral vessels as a result of haemorrhage can be examined. When healthy individuals are subjected to a venesection, e.g. blood donors, fainting may occur. Increase in the volume of the venesection raises the incidence of fainting, so much so that removal of I litre of blood produces fainting in almost every subject. It is, therefore, highly likely that fainting is an invariable consequence of haemorrhage if it is of sufficient volume. Fainting, or the vasovagal syndrome as it was called by Sir Thomas
Lewis, is a phenomenon which appears to be peculiar to man. At any rate there is as yet no definite evidence of a similar reaction in animals. Admittedly the reactions of animals to haemorrhage are usually examined under an anaesthetic, but fainting can take place under anaesthesia in man. Plethysmographic examination showed that a marked vasodilatation occurred in the muscle vessels at the time of the faint, a somewhat surprising finding. A full discussion of these findings has recently been published elsewhere (Barcroft and Edholm, 1946). ${ }^{3}$ The phenomenon is mentioned here to illustrate the fact that the circulatory changes in man and animals are not necessarily the same, and to provide one more example to show that studies in applied physiology frequently provide problems of fundamental interest.

There are some other striking differences between man and experimental animals in regard to haemorrhage. The rate of dilution of blood is very variable in man but is invariably much slower than has been found in animal work. After bleeding a dog one fifth or one quarter of its blood volume, the haematocrit rapidly falls indicating an increase in the volume of plasma due to dilution with tissue fluid. Blood volume measurements show that the original blood volume is restored very rapidly by this dilution, in a few hours at the most. In man this may take $24-48$ hours. This is one line of evidence indicating that the role of the spleen and other blood depots in man is far less prominent than in dogs or cats.

The best known effects of haemorrhage, the cardinal signs are an increased pulse rate and pallor. Both these have been extensively studied, but there are still several outstanding problems. The increased heart rate is usually explained as a consequence of the fall in blood pressure affecting the pressoreceptors in the carotid sinus and the aorta. But the increase in heart rate is out of all proportion to the fall in blood pressure. Haemorrhage in man has to be considerable before a significant fall in blood pressure takes place (apart from the changes due to fainting). So it is highly unlikely that the arterial pressure change is the essential factor. On the other hand it is possible that the fall in right auricular pressure is responsible. The opposite effect is well 
known as the Bainbridge reflex, i.e. a rise in pressure in the right auricle causes a reflex increase in heart rate, but an increased heart rate due to a fall in pressure here has not yet been clearly demonstrated.

The pallor following haemorrhage is usually considered to be a part of the peripheral vasoconstriction, which is generally accepted as one of the most important mechanisms compensating for the diminished blood volume. However, in man, moderate haemorrhage up to I litre does not appear to alter the rate of blood flow through the forearm. And yet there must be vasoconstriction somewhere, as the blood pressure is maintained in spite of the fall in cardiac output. The surprising fact is that this vasoconstriction is not where one might expect it to be, in the muscle vessels. Provisionally the splanchnic vessels must be chosen, as it is not yet possible to investigate these in man, and therefore this conclusion cannot be contradicted. It is, of course, possible that after more severe haemorrhage in man that the muscle vessels constrict also, but this has not been proved.

What practical value is there in these findings ? They may be of some assistance in the investigation of traumatic shock, but there is one point of immediate clinical interest. The heart rate is increased in haemorrhage and in assessing the extent of the blood loss the rapidity of the pulse is taken into consideration. If the subject has had a vasovagal attack the pulse rate slows markedly during the attack and following recovery does not increase again to the level before the attack. So the pulse rate may be deceptively slow even after severe haemorrhage.

There has been a considerable increase in our knowledge of the effects of haemorrhage in man during the war years. In spite of, or rather because of this new information it is realized that there are very big gaps still to be filled in.

The separate study of cardiac output and peripheral blood flow has provided, and will continue to provide, many valuable data. It has also been found useful to measure cardiac changes and peripheral reactions simultaneously. Such combined operations make it easier to assess the total vascular pattern. In particular the profound effect of cardiac changes on the peripheral circulation and vice versa has become obvious. In some cases of osteitis deformans (Paget's disease) dyspnoea, oedema and a very high pulse pressure suggest the diagnosis of cardiac failure due to aortic incompetence. ${ }^{5}$ More detailed examination has shown that there is no organic lesion of the heart in these cases. The cardiac output is high and the venous pressure is raised. The explanation of the apparent cardiac failure is to be found not in the heart, but in the peripheral circulation. Examination of the peripheral blood flow showed that there was an enormously increased blood flow through the affected bones, an increase that was estimated to be 'twenty-fold. When the disease is extensive, the bone flow alone may amount to several litres per minute, and a condition exists which is similar to a series of arteriovenous shunts. This accounts for the increased venous pressure and raised cardiac output. If these are maintained at high levels for long, cardiac failure may ensue.

A somewhat similar state of affairs is found in cases of traumatic arteriovenous aneurysm. When large vessels are involved and a considerable volume of blood is shunting through from artery to vein, the venous pressure is raised and so is the cardiac output. Such patients may also show signs of cardiac failure, although there is no cardiac lesion. These two examples demonstrate the effect of peripheral vascular disorders on the function of the heart. There are also many examples of changes within the heart altering the peripheral circulation. Plethysmograph recording of the peripheral blood flow in certain cases of left heart failure has shown an intense peripheral vasoconstriction. When venesection is performed, the peripheral blood flow increases considerably, although blood pressure may fall. In these cases the right auricular pressure is high and it is probably raised in the pulmonary vessels and the left auricle also. A raised right auricular pressure alone does not appear to be associated with peripheral vasoconstriction, but a raised pressure in the left auricle or pulmonary vessels may be responsible for a reflex peripheral vasoconstriction. Considerable work will be needed both to confirm this finding and to decide where the pressoreceptors concerned 
are sited. Other examples could be quoted to show that there are very frequently marked peripheral reactions to cardiac disease.

It is common practice both in physiology and in medicine to study the body systematically. This is both necessary and inevitable considering the degree of complexity of the body. In treating the cardiovascular system, the heart and the peripheral circulation are also considered separately. Again this is to a degree inevitable but the result is that cardiac disease is dealt with entirely apart from the state of the blood vessels and vice versa. So it is extremely important to stress the obvious, the physiological unity of the cardiovascular system. This is equally true when methods of treatment are under review. In recent publications $^{6}$ it has been shown that part of the action of digitalis is exerted peripherally. This is shown by the reduction of right auricular pressure by digitalis in cases of cardiac failure. It is suggested that digitalis mav act as a vasodilator. If this is confirmed it would indicate that treatment of the peripheral vascular system is as important in cardiac disease as treatment directly applied to the heart.

It has been pointed out above that there may well be cardiac effects on the circulation and vice versa. Such effects are probably exerted by reflex paths. It may be of some interest to consider some of the recent work on the vasomotor supply of peripheral blood vessels. Much of this has been done on patients who have been subjected to a sympathectomy. All blood vessels are supplied with vasomotor nerves, but the arterioles probably have the richest innervation. Both vasoconstrictor and vasodilator nerves are found. The former predominate in the periphery, in fact some would claim that vasodilator nerves have not been shown to exist in man in the vasomoto: supply of skin and muscle blood vessels. The arteriovenous anastamoses or shunts which are found particularly in the skin of the hands and feet, seceive a very rich vasomotor innervation. The sate of blood flow through the skin is greatly increased when these shunts are open; they provide an extra mechanism in addition to vasodilatation for increasing the rate of blood flow. In the hands and feet where these shunts are numerous there is a large range of blood flow. When the vessels are fully dilated the blood flow is at least one hundred times greater than the minimal blood flow. There is a continunus variation in the state of the blood vessels of the fingers, largely due to alterations in vasomotor tone. Considerable changes in blood flow can be evoked by quite trivial stimuli, a pinch, a sudden noise. A painful stimulus to any part of the body is followed by vasoconstriction of the fingers, which is due to vasomotor impulses. The circulation here is extremely labile and, owing to the rich vasomotor supply, is sensitive to many changes occurring elsewhere in the body. After sympathectomy these reflex alterations in blood flow are no longer obtained. Only local and humoral agencies can alter the circulation.

Muscle blood vessels on the other hand are more sensitive to humoral and physical stimuli than to vasomotor effects. However these vessels do receive vasoconstrictor nerves and there is evidence that they have a vasodilator nerve supply as well.

Under ordinary conditions of life the state of the blood vessels in the extremities varies continually. Sympathectomy removes this variable character, so the rate of blood flow is much more constant. The main effect of sympathectomy is the abolition of vasoconstrictor tone, so an immediate dilatation and an increased blood flow results. This vasodilatation is thought to be responsible for the success of the procedure. The increased blood flow removes products of metabolism which may have accumulated and be responsible amongst other things for pain. If excessive vasoconstrictor tone were responsible for peripheral vascular insufficiency, sympathectomy would undoubtedly be the rational treatment. At present evidence of such a condition as a dominant factor in peripheral vascular disease is not convincing. That does not mean such a condition does not exist, but it has not been demonstrated. However when there is evidence of vasospasm as in Reynaud's disease sympathectomy is frequently very successful. It is difficult to explain this success. The increased blood flow after sympathectomy does not persist. The denervated blood vessels recover or partially 
recover their tone within a few weeks. In the forearm the resting blood flow may be increased threefold by sympathectomy but returns to the pre-operative level within $t$ ireefour weeks. In the hand the blood flow diminishes from the high post-operative level but the vessels do not as a general rule completely recover their tone so the hand flow remains at a higher level more or less permanently.

Two other conditions have been successfully treated by sympathectomy, intermittent claudication and hypertension. But our present knowledge is inadequate to explain these results. The pain of intermittent claudication is frequently relieved for long periods by sympathectomy, but in these cases the resting blood flow in the legs is not increased for more than a few weeks at the most. It is for this and other reasons that Leriche and others have postulated that afferent fibres are interrupted by sympathectomy. This view was vigorously opposed by Lewis, ${ }^{7}$ and there the matter rests. It is clear that more research is required to study the effects of sympathectomy, and also on the anatomy of the sympathetic. Sheehan and his colleagues have been working on the anatomical problems for some years. Their results will undoubtedly be of the greatest importance in this respect. Similarly it is hard to understand the excellent results which have recently been described for the treatment of hypertension by extensive sympathetic denervation. A widespread vasodilatation could certainly reduce blood pressure, but the vessels recover their tone and the blood pressure still remains down.

It may be worth emphasizing now the immense value of direct work on man. In the first place the results obtained are directly applicable to clinical problems ; animal work, essential though it is, may sometimes be misleading. The vascular system of man differs in many ways from four-legged animals, presumably owing to man's upiight postu $e$. Several such examples have been given above.

In the second place the human subject is usually conscious and co-operative. Animal experiments in general are performed under anaesthesia, and this is a serious complicating factor. All anaesthetics produce changes in the circulation as, for instance, haemoconcentration. Failure to appreciate these effects has frequently invalidated the conclusions drawn from such experiments. On the other hand in human work it is essential to reduce emotional factors to a minimum. Procedures have to be carefully planned to obviate pain and discomfort. It was pointed out above that the peripheral circulation is remarkably labile, and markedly affected by emotional upsets or physical discomfort. This is also true of cardiac output. However, the subject can himself inform the observer about these factors, and so make it possible to eliminate them. These apparent disadvantages are really of value, as their removal is usually simple and the final experiment will be done under satisfactory conditions. The information obtained from the subject regarding sensations, etc., are also of the utmost value.

It could almost be claimed that the great extension of human experimentation in recent years, and particularly during the war, constitutes the main advance in physiology of clinical importance.

The chief conclusion that can be drawn from this cursory survey of recent work is that both physiologists and clinicians stand to gain from applied research. It is to be hoped that in future there will be far greater contact between the physiologist and the clinician. The physiologist will certainly profit by finding a host of problems, even though the clinician may not get his reward for some time.

\section{REFERENCES}

I. ABRAMSON, D. I. (1944), "Vascular responses in the extremities of man in health and disease," Chicago.

2. BARCLAY, A. E. FRANKLIN, $K$ J., and PRITCHARD, M. M. L. (1944), “The Foetal Circulation," Blackwell.

3. BARCROFT, H., and EDHOLM, O. G. (1946), Lancet, ii, 513.

4. COURNAND, A., and RANGES, H. A. (1941), Proc. Soc. exp. Biol. N.Y., 46, 462 .

5. EDHOLM, O. G., HOWARTH, S., and MCMICHAEL, J. (1945), Clin. Sci., 5, 249.

6. HOWARTH, S., MCMICHAEL, J., and SHARPEYSCHAFER, E.' P. (1946), Clin. Sci., 6, 41.

7. LEWIS, T. (1942), Pain, New York.

8. MCMICHAEL, J., and SHARPEY-SCHAFER, E. P. (1944), Brit. Heart. F., 6, 33 .

9. PATTERSON, S. W., and STARLING, E. H. (1914), 9. Physiol, 48, 357.

10. SHARPEY-SCHAFER, E. P. (1944), Clin. Sci., 5, 125. 